\title{
Screening for Key Pathways Associated with the Development of Osteoporosis by Bioinformatics Analysis
}

\author{
Yanqing Liu, ${ }^{1}$ Yueqiu Wang, ${ }^{2}$ Yanxia Zhang, ${ }^{3}$ Zhiyong Liu, ${ }^{4}$ Hongfei Xiang, \\ Xianbo Peng, ${ }^{6}$ Bohua Chen, ${ }^{5}$ and Guyou Jia ${ }^{2}$ \\ ${ }^{1}$ Department of Geriatric Medicine, Jining No. 1 People’s Hospital, Jining, Shandong 272011, China \\ ${ }^{2}$ Department of Joint Branch, Jining No. 2 People's Hospital, Jining, Shandong 272000, China \\ ${ }^{3}$ Department of Public Health, Jining Psychiatric Hospital, Jining, Shandong 272000, China \\ ${ }^{4}$ Department of Prevention and Health, Center for Disease Control and Prevention of Jining City, Jining, Shandong 272000, China \\ ${ }^{5}$ Department of Spine Surgery, The Affiliated Hospital of Qingdao University, Qingdao, Shandong 266003, China \\ ${ }^{6}$ Department of Orthopedics, Shandong Provincial Qianfoshan Hospital, Shandong University, Jinan, Shandong 250014, China
}

Correspondence should be addressed to Bohua Chen; chenbohual337@hotmail.com and Guyou Jia; jiaguyoul354@hotmail.com

Received 21 October 2016; Revised 13 January 2017; Accepted 26 January 2017; Published 30 March 2017

Academic Editor: Shinji Kuroda

Copyright (C) 2017 Yanqing Liu et al. This is an open access article distributed under the Creative Commons Attribution License, which permits unrestricted use, distribution, and reproduction in any medium, provided the original work is properly cited.

Objectives. We aimed to find the key pathways associated with the development of osteoporosis. Methods. We downloaded expression profile data of GSE35959 and analyzed the differentially expressed genes (DEGs) in 3 comparison groups (old_op versus middle, old_op versus old, and old_op versus senescent). KEGG (Kyoto Encyclopedia of Genes and Genomes) pathway enrichment analyses were carried out. Besides, Venn diagram analysis and gene functional interaction (FI) network analysis were performed. Results. Totally 520 DEGs, 966 DEGs, and 709 DEGs were obtained in old_op versus middle, old_op versus old, and old_op versus senescent groups, respectively. Lysosome pathway was the significantly enriched pathways enriched by intersection genes. The pathways enriched by subnetwork modules suggested that mitotic metaphase and anaphase and signaling by Rho GTPases in module 1 had more proteins from module. Conclusions. Lysosome pathway, mitotic metaphase and anaphase, and signaling by Rho GTPases may be involved in the development of osteoporosis. Furthermore, Rho GTPases may regulate the balance of bone resorption and bone formation via controlling osteoclast and osteoblast. These 3 pathways may be regarded as the treatment targets for osteoporosis.

\section{Introduction}

Primary osteoporosis is a polygenetic disease characterized by an imbalance of bone homeostasis including microarchitectural deteriorations and low bone mineral density [1]. It is reported that approximately 5.5 million men and 22 million women had osteoporosis in the European Union in 2010 [2]. Risk factors for osteoporosis include gender, advanced age, and diminished sex steroid production after menopause and in elderly individuals and so on $[3,4]$. Thus, it is important to get the molecular mechanisms for osteoporosis and then find the effective treatment methods for it.

It has been reported that strontium results in increased bone formation and decreased bone resorption by the modulation of several pathways including CaSR, ERK1/2-MAPK, and NFATc/Wnt signaling pathways [5]. One study showed that RANKL (receptor activator of NF- $\kappa$ B ligand)/RANK (receptor activator of NF- $\kappa \mathrm{B}) / \mathrm{OPG}$ (osteoprotegerin) signaling system was essential for skeletal homeostasis, and disruption of it led to inhibition of bone resorption in vitro [6]. The bone formation inhibitor sclerostin encoded by SOST binds in vitro to low density LRP5/6 (lipoprotein receptor-related protein) Wnt coreceptors, thereby inhibiting $\mathrm{Wnt} / \beta$-catenin signaling, a central pathway of skeletal homeostasis, and LRP5 deficiency results in OPPG (osteoporosis-pseudoglioma), whereas SOST deficiency induces lifelong bone gain in mice and humans [7]. Azuma et al. indicated that the SXR/ PXR (Nuclear Receptor Subfamily 1, Group I, Member 2) dependent signaling pathway could mediate the protective effects of vitamin $\mathrm{K}$ for bone [8]. Pineda et al. suggested 
that antioxidant pathways played important roles in bone homeostasis [9]. Furthermore, functional polymorphisms of the P2X7 (Purinergic Receptor P2X, Ligand Gated Ion Channel, 7) receptor gene are related to osteoporosis [10]. Two single nucleotide polymorphisms of rs4237 and rs2501431 in CNR2 (Cannabinoid Receptor 2) gene may result in postmenopausal osteoporosis in Han Chinese women [11]. Mettl21c (methyltransferase-like 21C) may play bone-muscle pleiotropic roles through the regulation of the NF- $\kappa$ B signaling pathway [12]. However, the underlying mechanisms or key regulating factors for osteoporosis are not fully understood.

Bone marrow mesenchymal cells (BMSCs) are the major source of osteoprogenitor cells resulting in remodeling of bone in adults [13]. The former researches using the data of GSE35959 demonstrated that nuclear factor I-C (NFI-C) regulated osteoblast differentiation [14], mechanical stimulation affected genes expression associated with osteogenic differentiation of BMSCs through the regulation of HDAC1 (Histone Deacetylase 1) [15], or the transcriptional profile of MSC populations in primary osteoporosis showed overexpression of osteogenic inhibitors [16]. In contrast to previous studies, we downloaded this data and analyzed the differentially expressed genes (DEGs) in 3 comparison groups. KEGG (Kyoto Encyclopedia of Genes and Genomes) pathway enrichment analyses were carried out. Besides, Venn diagram analysis and gene functional interaction (FI) network analysis were performed. We aimed to understand the key pathways associated with the development of osteoporosis and then find the effective treatment methods for it.

\section{Materials and Methods}

2.1. Expression Profile Data. The expression profile data of GSE35959 deposited by Benisch et al. was downloaded from the GEO (Gene Expression Omnibus, https://www.ncbi.nlm .nih.gov/geo/) database [16]. A total of 5 middle aged human mesenchymal stem cells (MSC) samples (middle), 4 old elderly MSC samples (old), 5 primary osteoporosis elderly MSC samples (old_op), and 5 senescent MSC samples (senescent) were included in this study. The data were based on the platform of GPL570 (Affymetrix Human Genome U133 Plus 2.0 Array, Affymetrix, Inc., Santa Clara, California, USA).

2.2. Data Preprocessing. The raw data were preprocessed by R package affy (version: 3.24.15) [17] in Bioconductor (http:// www.bioconductor.org/). Background correction, normalization between arrays, and calculated expression were included in the process of preprocessing. The probe ID was transformed into gene symbol combined with annotation files of the platform.

2.3. DEGs Analysis. Significant $p$ value for DEGs in primary osteoporosis elderly MSC versus middle aged MSC (old_op versus middle), primary osteoporosis elderly MSC versus old elderly MSC (old_op versus old), and primary osteoporosis elderly MSC versus senescent MSC (old_op versus senescent) were analyzed with $t$-test in limma (version: 3.24.15) [18]. The $p$ value was adjusted as FDR (false discovery rate) values by $\mathrm{BH}$ (Benjamini-Hochberg) [19]. FDR $<0.05$ and $|\log 2 \mathrm{FC}| \geq$ 1 were used as cut-off criterion for DEGs.
2.4. KEGG (Kyoto Encyclopedia of Genes and Genomes) Pathway Enrichment Analysis. KEGG is a database used for putting associated genes into the corresponding pathways [20]. R package clusterProfiler (version: 2.2.7) [21] based on KEGG.db annotation package was used to the pathway enrichment analysis. Significant $p$ values for enriched DEGs were calculated by hypergeometric distribution, and $p<0.05$ was set as significantly enriched pathway.

2.5. Venn Diagram Analysis for DEGs. VennPlex (http://www .irp.nia.nih.gov/bioinformatics/vennplex.html) [22] can be used to analyze Venn diagram for multiple dataset by using gene expression values and screen out the significant genes. Furthermore, it can display the number of intersection genes that are upregulated, downregulated, and contraregulated, respectively. By using a large set of functional association data including protein and genetic interactions, pathways, coexpression, colocalization, and protein domain similarity, GeneMANIA [23] can find other genes associated with a set of input genes.

DEGs and $\log 2 \mathrm{FC}$ of 3 comparison groups were input into VennPlex, and the similarities and differences in 3 comparison groups were observed. Furthermore, KEGG pathways enriched by intersection DEGs in 3 groups were obtained. The correlations among intersection DEGs were analyzed by Cytoscape (version: 3.2.1) [24] app-GeneMANIA.

2.6. Gene Functional Interaction (FI) Network Analysis. The ReactomeFIViz app [25] can construct FI network, calculate correlations (average Pearson correlation coefficient) among genes, use the calculated correlations as weights for edges, apply Monte Carlo localization graph clustering algorithm to the weighted FI network, and generate a subnetwork for a list of selected network modules.

Gene functional interaction networks were analyzed with Cytoscape app-ReactomeFIViz. The input dataset was expression matrix of all DEGs, and pathway enrichment analysis for every function module was performed to find biological pathway involved by every module genes (FDR < 0.05). Other ReactomeFI parameters were set as defaults.

\section{Results}

3.1. DEGs Analysis. A total of 520 DEGs, 966 DEGs, and 709 DEGs were obtained in old_op versus middle, old_op versus old, and old_op versus senescent groups, respectively (Table 1).

3.2. Functional Enrichment Analysis. The significantly enriched KEGG pathways for 3 comparison groups were shown in Figure 1. Cell cycle pathway was the significantly enriched pathway in old_op versus middle, and focal adhesion pathway was the significantly enriched pathway in old_op versus old, as well as old_op versus senescent.

3.3. Venn Diagram Analysis for DEGs. Venn diagram analysis for DEGs was shown in Figure 2. A total of 36 upregulated genes and 47 downregulated genes were included in DEGs of 3 comparison groups. KEGG pathways significantly enriched 
TABLE 1: The number of differentially expressed genes (DEGs) for 3 comparison groups.

\begin{tabular}{lccc}
\hline Group & Upregulated gene count & Downregulated gene count & Total \\
\hline old_op versus middle & 91 & 429 & 520 \\
old_op versus old & 270 & 696 & 966 \\
old_op versus senescent & 336 & 373 & 709 \\
\hline
\end{tabular}

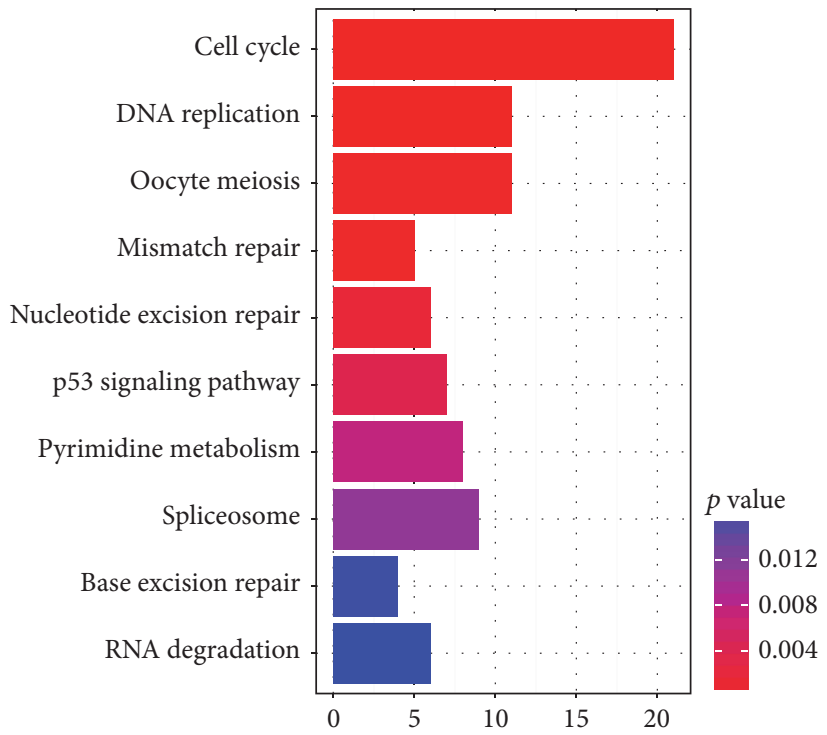

(a)

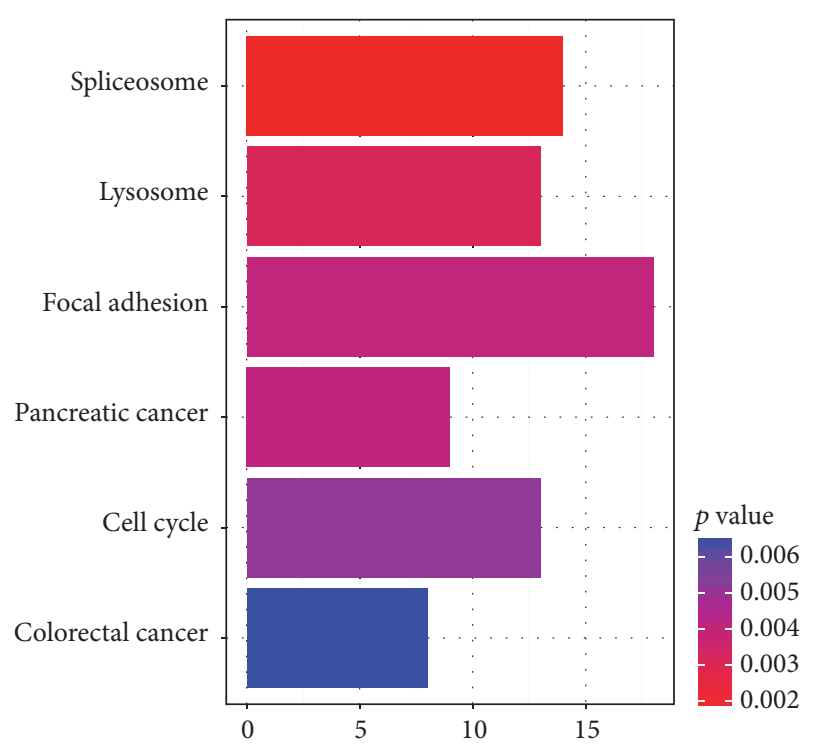

(b)

ECM-receptor interaction

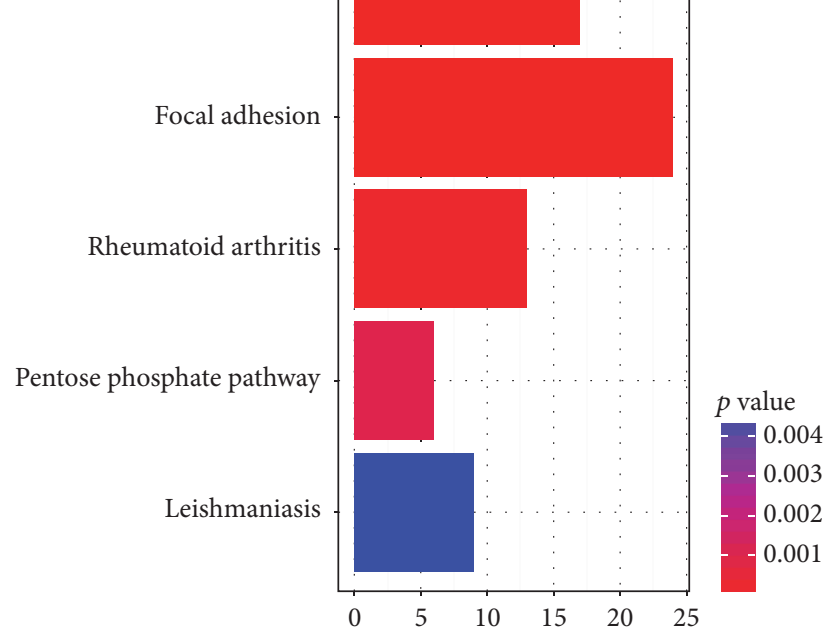

(c)

FIGURE 1: The significantly enriched KEGG (Kyoto Encyclopedia of Genes and Genomes) pathways for 3 comparison groups. (a) Primary osteoporosis elderly MSC versus middle aged MSC; (b) primary osteoporosis elderly MSC versus old elderly MSC; (c) primary osteoporosis elderly MSC versus senescent MSC.

by intersection genes were shown in Figure 3. Lysosome pathway enriched by LAPTM5 (Lysosomal Protein Transmembrane 5, upregulated), CTSD (Cathepsin D, upregulated), LIPA (Lipase A, Lysosomal Acid Type, downregulated), and
AGA (Aspartylglucosaminidase, downregulated) was the significantly enriched pathways enriched by intersection genes. The interaction network among intersection genes obtained by GeneMANIA analysis was shown in Figure 4, and 79 


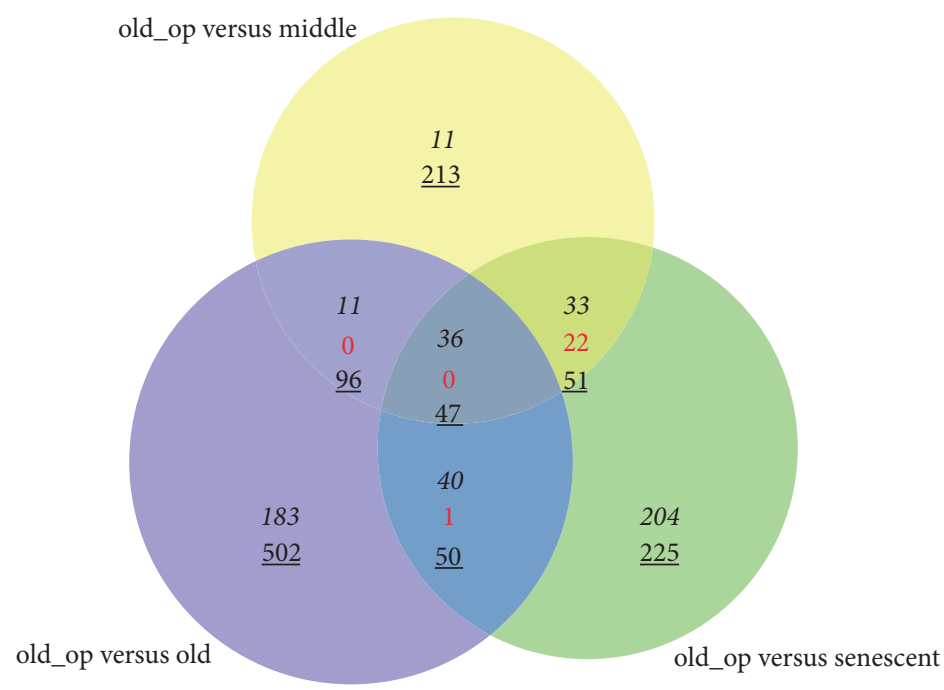

FIGURE 2: Venn diagram analysis for differentially expressed genes (DEGs).

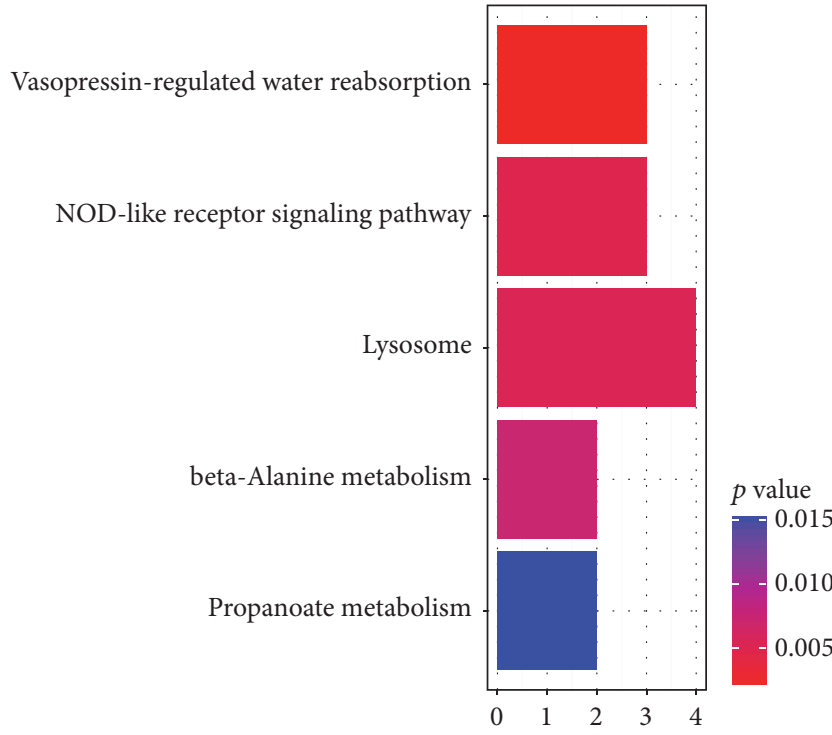

Figure 3: KEGG (Kyoto Encyclopedia of Genes and Genomes) pathways significantly enriched by intersection genes.

intersection genes and 367 interaction pairs were included in the interaction network. In addition, this interaction network included 5 association data items (262 coexpressions, 7 physical interactions, 80 genetic interactions, 17 colocalization, and 1 pathway).

3.4. Gene Functional Interaction Network Analysis. The functional interaction network for DEGs obtained by ReactomeFI was shown in Figure 5, and 240 nodes and 1309 interaction pairs were included in it. Furthermore, this network included 16 significant subnetwork modules (Table 2). Module 0 and module 1 had more nodes. The absolute value of average correlation between subnetwork module genes was high. In addition, pathways enriched by subnetwork modules were
TABLE 2: The information of 16 significant subnetwork modules.

\begin{tabular}{lcc}
\hline Module & Nodes in module & Average correlation \\
\hline 0 & 51 & 0.5639 \\
1 & 37 & 0.7701 \\
2 & 24 & 0.6355 \\
3 & 14 & 0.848 \\
4 & 14 & 0.7763 \\
5 & 14 & 0.5407 \\
6 & 10 & 0.5594 \\
7 & 10 & 0.4676 \\
8 & 9 & 0.7657 \\
9 & 9 & 0.7226 \\
10 & 9 & 0.5833 \\
11 & 9 & 0.5608 \\
12 & 9 & 0.5231 \\
13 & 9 & 0.3758 \\
14 & 8 & 0.3205 \\
15 & 7 & 0.546 \\
\hline
\end{tabular}

shown in Table 3. Mitotic metaphase and anaphase and signaling by Rho GTPases in module 1 had more proteins from module.

\section{Discussions}

In the current study, with the expression profile data of GSE35959, totally 520 DEGs, 966 DEGs, and 709 DEGs were obtained in old_op versus middle, old_op versus old, and old_op versus senescent groups, respectively. Lysosome pathway was the significantly enriched pathways enriched by intersection genes. Furthermore, the pathways enriched by subnetwork modules suggested that mitotic metaphase and anaphase and signaling by Rho GTPases in module 1 had more proteins from module. 


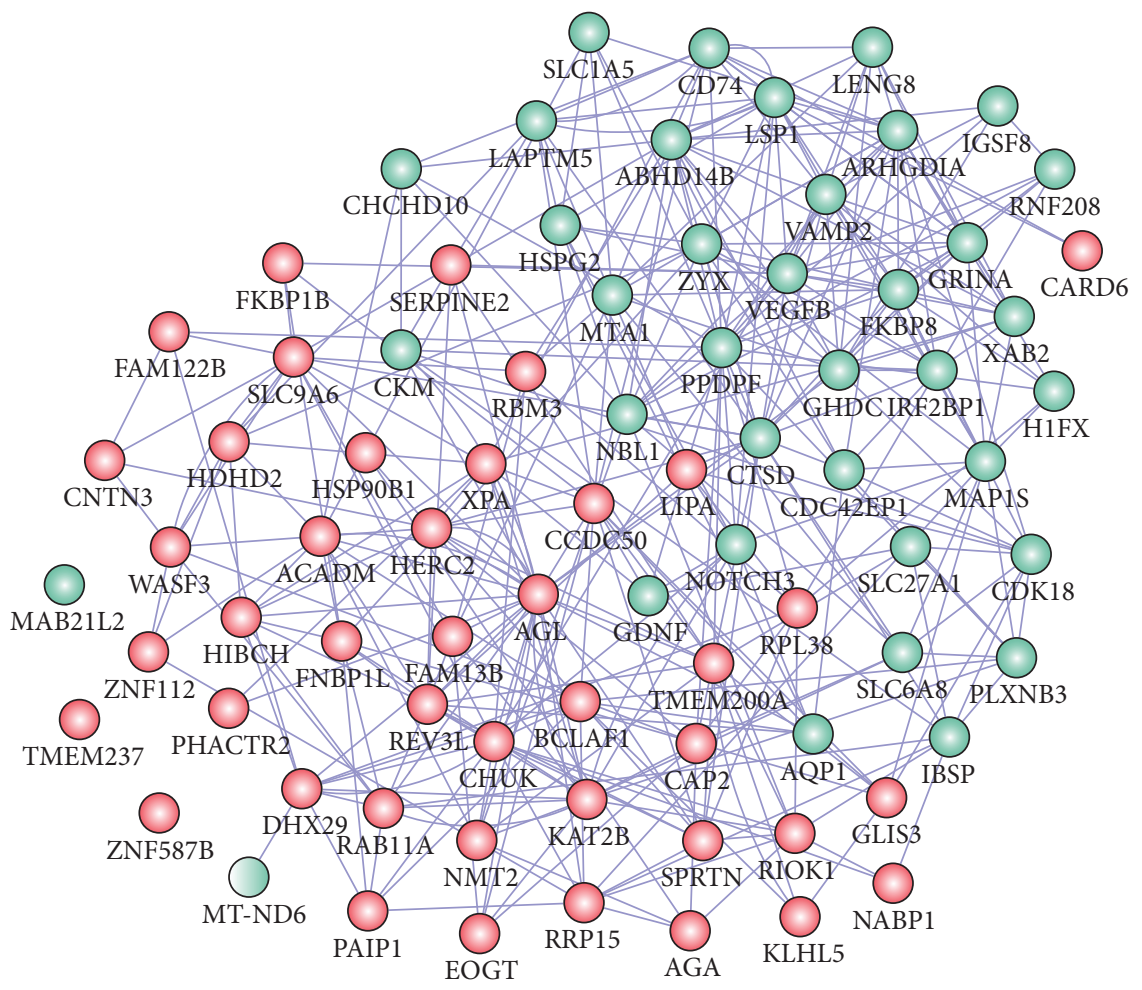

FIGURE 4: The interaction network among intersection genes. Red nodes: downregulated; green nodes: upregulated.

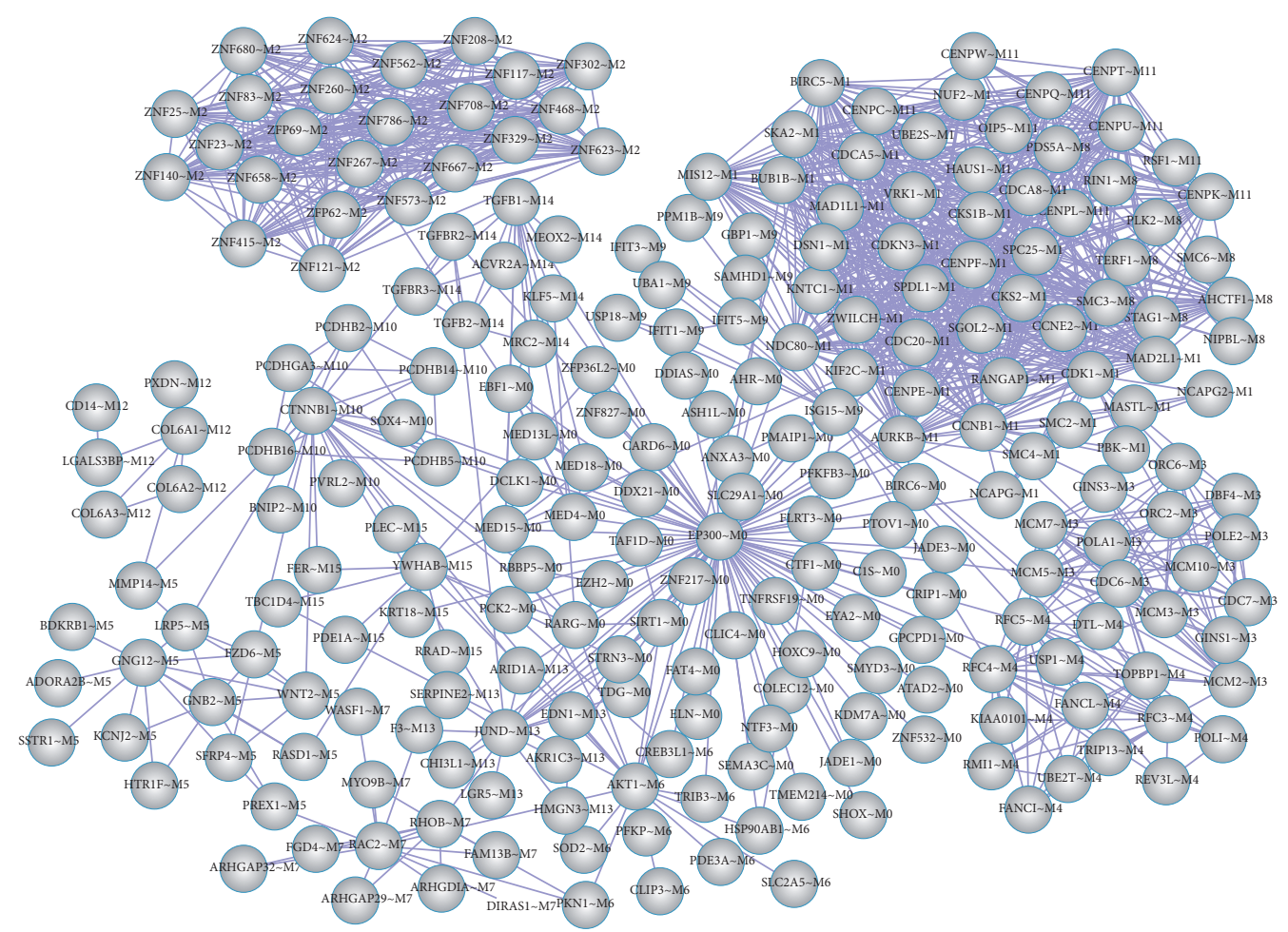

FIGURE 5: The functional interaction network for differentially expressed genes (DEGs); "gene symbol M0-15" represents the name of nodes; suffix "M0-15" represents the subnetwork modules that this gene belongs to. 
TABLE 3: Pathways enriched by subnetwork modules.

\begin{tabular}{|c|c|c|c|}
\hline Module & Gene set & Protein from module & FDR \\
\hline 0 & Transcriptional regulation of white adipocyte differentiation (R) & 6 & $2.72 E-05$ \\
\hline 0 & Chromatin modifying enzymes $(\mathrm{R})$ & 6 & $2.03 E-03$ \\
\hline 1 & Mitotic metaphase and anaphase $(\mathrm{R})$ & 23 & $2.00 E-15$ \\
\hline 1 & Signaling by Rho GTPases (R) & 21 & $2.00 E-15$ \\
\hline 3 & Synthesis of DNA (R) & 11 & $4.44 E-16$ \\
\hline 3 & S phase $(\mathrm{R})$ & 11 & $4.44 E-16$ \\
\hline 4 & DNA damage bypass $(\mathrm{R})$ & 8 & $9.99 E-16$ \\
\hline 4 & Fanconi anemia pathway $(\mathrm{N})$ & 9 & $9.99 E-16$ \\
\hline 5 & Heterotrimeric G-protein signaling pathway-Gq alpha and Go alpha mediated pathway $(\mathrm{P})$ & 5 & $1.74 E-05$ \\
\hline 5 & Wnt signaling pathway $(\mathrm{P})$ & 6 & $2.86 E-05$ \\
\hline 6 & Protein kinase a at the centrosome (B) & 2 & $6.92 E-03$ \\
\hline 6 & Prostate cancer $(\mathrm{K})$ & 3 & $6.92 E-03$ \\
\hline 7 & Signaling by Rho GTPases (R) & 8 & $4.73 E-10$ \\
\hline 7 & Regulation of RhoA activity (N) & 2 & $1.07 E-02$ \\
\hline 8 & Mitotic telophase/cytokinesis (R) & 4 & $7.08 E-09$ \\
\hline 8 & Mitotic prometaphase (R) & 4 & $8.56 E-06$ \\
\hline 9 & Interferon alpha/beta signaling $(\mathrm{R})$ & 5 & $6.83 E-09$ \\
\hline 9 & ISG15 antiviral mechanism (R) & 4 & $6.35 E-07$ \\
\hline 10 & Cadherin signaling pathway $(\mathrm{P})$ & 6 & $4.47 E-09$ \\
\hline 10 & Wnt signaling pathway $(\mathrm{P})$ & 6 & $7.94 E-07$ \\
\hline 11 & Nucleosome assembly (R) & 9 & $5.55 E-16$ \\
\hline 11 & Mitotic prometaphase $(\mathrm{R})$ & 6 & $1.36 E-10$ \\
\hline 12 & Betal integrin cell surface interactions $(\mathrm{N})$ & 4 & $4.71 E-08$ \\
\hline 12 & ECM-receptor interaction $(\mathrm{K})$ & 3 & $2.45 E-05$ \\
\hline 13 & Formation of fibrin clot (clotting cascade) (R) & 2 & $1.36 E-02$ \\
\hline 13 & AP-1 transcription factor network $(\mathrm{N})$ & 2 & $2.12 E-02$ \\
\hline 14 & TGF-beta signaling pathway (P) & 4 & $4.87 E-06$ \\
\hline 14 & TGF-beta signaling pathway (K) & 4 & $4.87 E-06$ \\
\hline 15 & Insulin-mediated glucose transport $(\mathrm{N})$ & 2 & $9.27 E-03$ \\
\hline 15 & Class I PI3K signaling events mediated by Akt (N) & 2 & $9.27 E-03$ \\
\hline
\end{tabular}

The source of pathway database: C: CellMap, R: Reactome, K: KEGG, N: NCI PID, P: Panther, and B: BioCarta.

Lysosome pathway was the significantly enriched pathways enriched by intersection genes in our present study. RANKL/RANK/OPG signaling system was essential for skeletal homeostasis, and one study showed that RANKL was found to be localized to secretory lysosomes in osteoblastic cells [26]. Yoneshima et al. suggested that lysosomal biogenesis mediated by TFEB (Transcription Factor EB) might be involved in osteoblast differentiation [27]. Furthermore, osteoporosis is characterized by an imbalance of bone resorption and bone formation [28]. Thus, our results are according to the previous studies and show that lysosome pathway plays important parts in the development of osteoporosis.

Furthermore, mitotic metaphase and anaphase in module 1 had more proteins from module in this study. The transcription of Runx 2 mRNA is dependent on mitosis and the translation of it after mitosis in early osteoprogenitors to control the gene expression required for reinforcement of cell fate decisions in committed preosteoblasts [29]. One study indicated that LRP1 (low density lipoprotein receptor-related protein 1) could activate the p42/44 MAPK (mitogen-activated protein kinase) pathway and then lead to the mitosis of osteoblasts [30]. Thus, mitosis is significant for the osteoblasts. Besides, osteoblasts play key roles in the bone formation, and osteoporosis is characterized by the imbalance of bone resorption and bone formation. Therefore, combined with our results, we speculate that mitotic metaphase and anaphase may play key roles in the progression of osteoporosis.

In addition, our study also showed that signaling by Rho GTPases in module 1 had more proteins from module. Rho GTPases may control podosome arrangements in osteoclasts [31]. Brazier et al. showed that the Rho GTPase Wrch1 (Ras Homolog Family Member U) regulated precursor adhesion and migration of osteoclast [32]. Touaitahuata et al. suggested that Rho GTPases could modulate osteoclast differentiation and bone resorption [33]. Besides, Wan et al. indicated that Rho GTPases controlled TCF/LEF (hepatocyte nuclear factor 4 , alpha) activity and nuclear localization of $\beta$-catenin in osteoblasts under flow [34]. Thus, Rho GTPases may play roles in osteoclast and osteoblast. Rho GTPases may regulate the balance of bone resorption and bone formation via 
controlling osteoclast and osteoblast. Combined with our results, we think that signaling by Rho GTPases may be involved in the development of osteoporosis.

However, there are several limitations in this study. First, only 19 samples including 5 middle aged MSCs, 4 old elderly MSCs, 5 primary osteoporosis elderly MSCs, and 5 senescent MSCs were included in this study. Second, in Results, the interaction network among intersection genes includes 79 intersection genes and 367 interaction pairs, but there is no significant difference among the weight of these intersection genes. Third, our study is concluded from the bioinformatics analysis of the expression profile data downloaded from the GEO database, and further experiments are needed to verify our results.

\section{Conclusions}

In conclusion, lysosome pathway, mitotic metaphase and anaphase, and signaling by Rho GTPases may be involved in the development of osteoporosis. Furthermore, Rho GTPases may regulate the balance of bone resorption and bone formation via controlling osteoclast and osteoblast. Lysosome pathway, mitotic metaphase and anaphase, and signaling by Rho GTPases may be regarded as the treatment targets for osteoporosis. However, further studies with large samples and verification experiments are needed.

\section{Additional Points}

Highlights. (1) Lysosome pathway may be involved in the development of osteoporosis. (2) Mitotic metaphase and anaphase may play key roles in osteoporosis. (3) Signaling by Rho GTPases may be important for the development of osteoporosis. (4) These 3 pathways may be regarded as the treatment targets for osteoporosis.

\section{Competing Interests}

The authors declare that they have no competing interests.

\section{Authors' Contributions}

Yanqing Liu and Yueqiu Wang should be regarded as co-first authors.

\section{References}

[1] T. D. Rachner, S. Khosla, and L. C. Hofbauer, "Osteoporosis: now and the future," The Lancet, vol. 377, no. 9773, pp. 12761287, 2011.

[2] A. Svedbom, E. Hernlund, M. Ivergård et al., "Osteoporosis in the European Union: a compendium of country-specific reports," Archives of Osteoporosis, vol. 8, no. 1-2, pp. 1-218, 2013.

[3] E. Seeman, "Bone quality: the material and structural basis of bone strength," Journal of Bone and Mineral Metabolism, vol. 26, no. 1, pp. 1-8, 2008.

[4] P. Pietschmann, M. Rauner, W. Sipos, and K. Kerschan-Schindl, "Osteoporosis: an age-related and gender-specific disease-a mini-review," Gerontology, vol. 55, no. 1, pp. 3-12, 2009.
[5] Z. Saidak and P. J. Marie, "Strontium signaling: molecular mechanisms and therapeutic implications in osteoporosis," Pharmacology \& Therapeutics, vol. 136, no. 2, pp. 216-226, 2012.

[6] B. F. Boyce and L. Xing, "Functions of RANKL/RANK/OPG in bone modeling and remodeling," Archives of Biochemistry and Biophysics, vol. 473, no. 2, pp. 139-146, 2008.

[7] M.-K. Chang, I. Kramer, H. Keller et al., "Reversing LRP 5dependent osteoporosis and SOST deficiency-induced sclerosing bone disorders by altering WNT signaling activity," Journal of Bone and Mineral Research, vol. 29, no. 1, pp. 29-42, 2014.

[8] K. Azuma, Y. Ouchi, and S. Inoue, "Vitamin K: novel molecular mechanisms of action and its roles in osteoporosis," Geriatrics \& Gerontology International, vol. 14, no. 1, pp. 1-7, 2014.

[9] B. Pineda, E. Serna, A. Laguna-Fernández et al., "Gene expression profile induced by ovariectomy in bone marrow of mice: a functional approach to identify new candidate genes associated to osteoporosis risk in women," Bone, vol. 65, pp. 33-41, 2014.

[10] L. Husted, T. Harsløf, L. Stenkjær, M. Carstens, N. Jørgensen, and B. L. Langdahl, "Functional polymorphisms in the P2X7 receptor gene are associated with osteoporosis," Osteoporosis International, vol. 24, no. 3, pp. 949-959, 2013.

[11] C. Zhang, J. Ma, G. Chen, D. Fu, L. Li, and M. Li, "Evaluation of common variants in CNR2 gene for bone mineral density and osteoporosis susceptibility in postmenopausal women of Han Chinese," Osteoporosis International, vol. 26, no. 12, pp. $2803-$ 2810, 2015.

[12] J. Huang, Y. H. Hsu, C. Mo et al., "METTL21C is a potential pleiotropic gene for osteoporosis and sarcopenia acting through the modulation of the NF- $\kappa \mathrm{B}$ signaling pathway," Journal of Bone and Mineral Research, vol. 29, no. 7, pp. 1531-1540, 2014.

[13] H.-M. Hu, L. Yang, Z. Wang et al., "Overexpression of integrin a2 promotes osteogenic differentiation of hBMSCs from senile osteoporosis through the ERK pathway," International Journal of Clinical and Experimental Pathology, vol. 6, no. 5, pp. 841-852, 2013.

[14] D. S. Lee, H. W. Choung, H. J. Kim et al., "NFI-C regulates osteoblast differentiation via control of osterix expression," Stem cells, vol. 32, no. 9, pp. 2467-2479, 2014.

[15] J. Wang, C. Wang, N. Zhang et al., "Mechanical stimulation orchestrates the osteogenic differentiation of human bone marrow stromal cells by regulating HDACl," Cell Death \& Disease, vol. 7, no. 5, article e2221, 2016.

[16] P. Benisch, T. Schilling, L. Klein-Hitpass et al., "The transcriptional profile of mesenchymal stem cell populations in primary osteoporosis is distinct and shows overexpression of osteogenic inhibitors," PLoS ONE, vol. 7, no. 9, Article ID e45142, 2012.

[17] L. Gautier, L. Cope, B. M. Bolstad, and R. A. Irizarry, "Affyanalysis of Affymetrix GeneChip data at the probe level," Bioinformatics, vol. 20, no. 3, pp. 307-315, 2004.

[18] G. K. Smyth, "Limma: linear models for microarray data," in Bioinformatics and Computational Biology Solutions Using R and Bioconductor, pp. 397-420, Springer, Berlin, Germany, 2005.

[19] J. A. Ferreira, “The Benjamini-Hochberg method in the case of discrete test statistics," International Journal of Biostatistics, vol. 3, no. 1, 2007.

[20] E. Altermann and T. R. Klaenhammer, "PathwayVoyager: pathway mapping using the Kyoto Encyclopedia of Genes and Genomes (KEGG) database," BMC Genomics, vol. 6, no. 1, article 60, 2005.

[21] G. Yu, L.-G. Wang, Y. Han, and Q.-Y. He, "clusterProfiler: an R package for comparing biological themes among gene clusters," 
Omics: A Journal of Integrative Biology, vol. 16, no. 5, pp. 284287, 2012.

[22] H. Cai, H. Chen, T. Yi et al., "VennPlex-a novel Venn diagram program for comparing and visualizing datasets with differentially regulated datapoints," PLoS ONE, vol. 8, no. 1, Article ID e53388, 2013.

[23] J. Montojo, K. Zuberi, H. Rodriguez, G. D. Bader, and Q. Morris, "GeneMANIA: fast gene network construction and function prediction for Cytoscape," F1000Research, vol. 3, article 153, 2014.

[24] P. Shannon, A. Markiel, O. Ozier et al., "Cytoscape: a software environment for integrated models of biomolecular interaction networks," Genome Research, vol. 13, no. 11, pp. 2498-2504, 2003.

[25] G. Wu, E. Dawson, A. Duong, R. Haw, and L. Stein, "ReactomeFIViz: a Cytoscape app for pathway and network-based data analysis," F1000Research, vol. 3, article 146, 2014.

[26] Y. Kariya, M. Honma, A. Hanamura et al., "Rab27a and Rab27b are involved in stimulation-dependent RANKL release from secretory lysosomes in osteoblastic cells," Journal of Bone and Mineral Research, vol. 26, no. 4, pp. 689-703, 2011.

[27] E. Yoneshima, K. Okamoto, E. Sakai, K. Nishishita, N. Yoshida, and T. Tsukuba, "The transcription factor EB (TFEB) regulates osteoblast differentiation through ATF4/CHOP-dependent pathway," Journal of Cellular Physiology, vol. 231, no. 6, pp. 1321-1333, 2016.

[28] N. M. Appelman-Dijkstra and S. E. Papapoulos, "Modulating bone resorption and bone formation in opposite directions in the treatment of postmenopausal osteoporosis," Drugs, vol. 75, no. 10, pp. 1049-1058, 2015.

[29] N. Varela, A. Aranguiz, C. Lizama et al., "Mitotic inheritance of mRNA facilitates translational activation of the osteogeniclineage commitment factor Runx2 in progeny of osteoblastic cells," Journal of Cellular Physiology, vol. 231, no. 5, pp. 1001-1014, 2016.

[30] J.-M. Hou, E.-Y. Chen, F. Lin et al., "Lactoferrin induces osteoblast growth through IGF-1R," International Journal of Endocrinology, vol. 2015, Article ID 282806, 9 pages, 2015.

[31] S. Ory, H. Brazier, G. Pawlak, and A. Blangy, "Rho GTPases in osteoclasts: orchestrators of podosome arrangement," European Journal of Cell Biology, vol. 87, no. 8-9, pp. 469-477, 2008.

[32] H. Brazier, G. Pawlak, V. Vives, and A. Blangy, "The Rho GTPase Wrchl regulates osteoclast precursor adhesion and migration," The International Journal of Biochemistry \& Cell Biology, vol. 41, no. 6, pp. 1391-1401, 2009.

[33] H. Touaitahuata, A. Blangy, and V. Vives, "Modulation of osteoclast differentiation and bone resorption by Rho GTPases," Small GTPases, vol. 5, no. 1, article e28119, 2014.

[34] Q. Wan, E. Cho, P. Zhang, H. Yokota, and S. Na, "Rho GTPases control nuclear localization of beta-catenin and TCF/LEF activity in osteoblasts under flow," Journal of Bone and Mineral Research, vol. 28, 2013. 

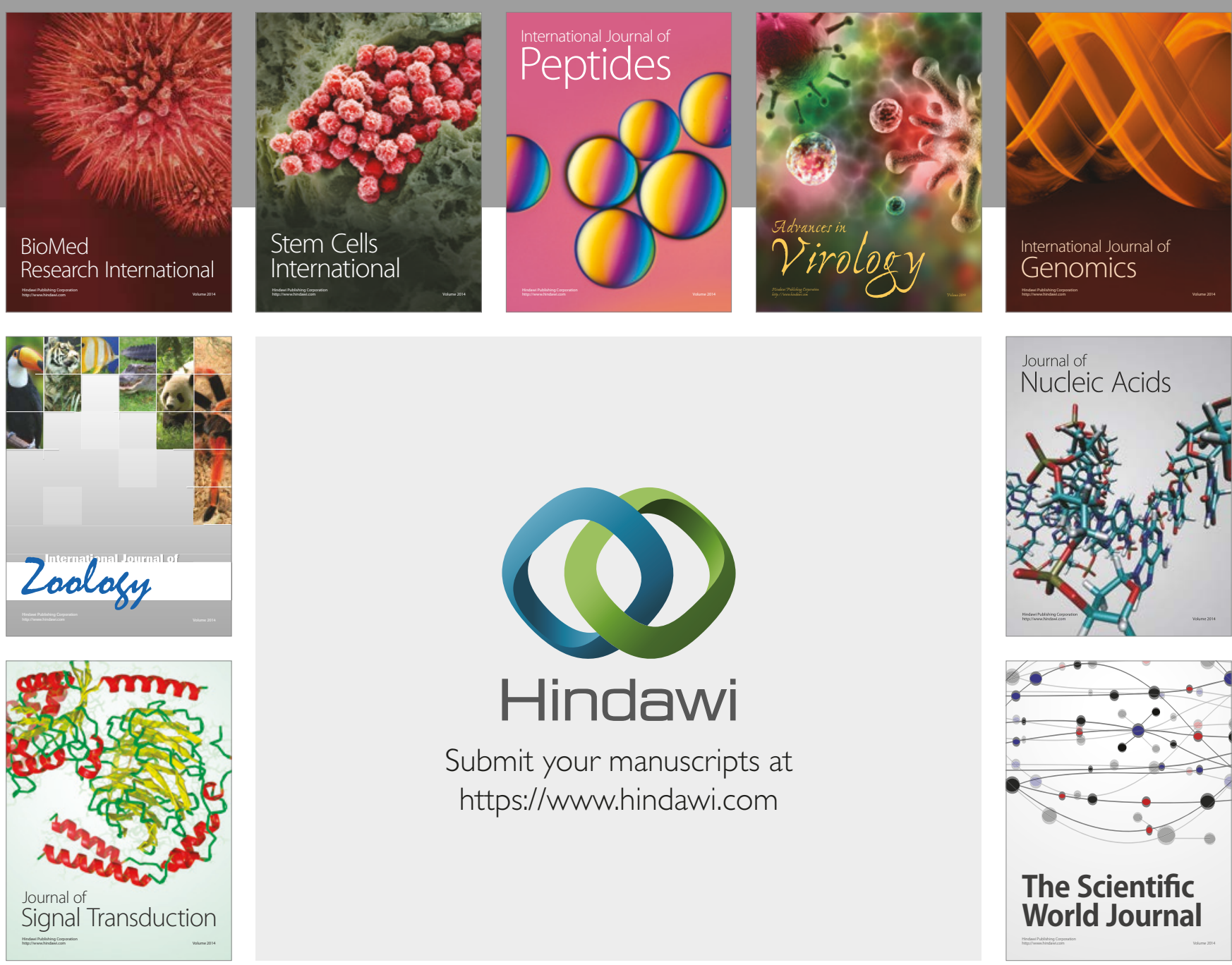

Submit your manuscripts at

https://www.hindawi.com
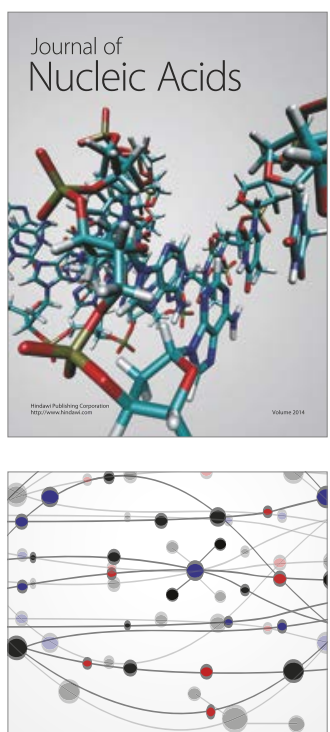

The Scientific World Journal

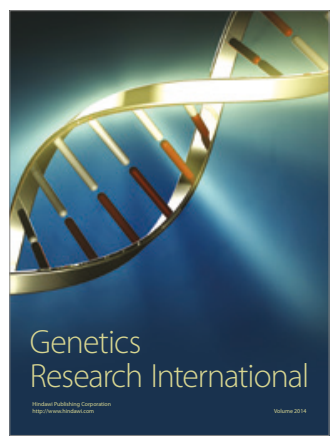

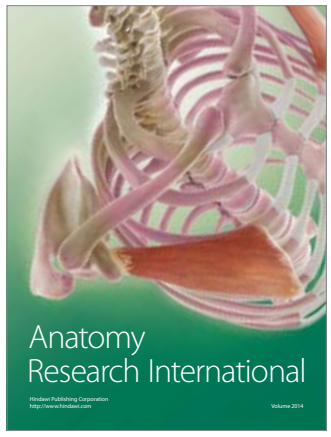

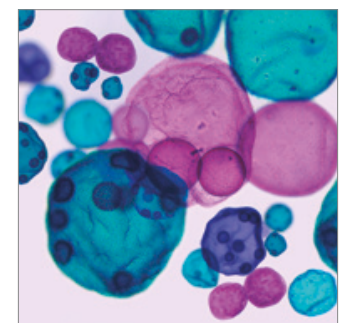

International Journal of Microbiology
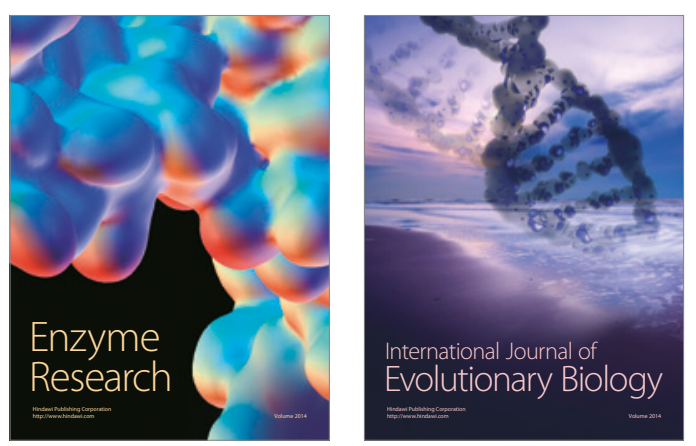
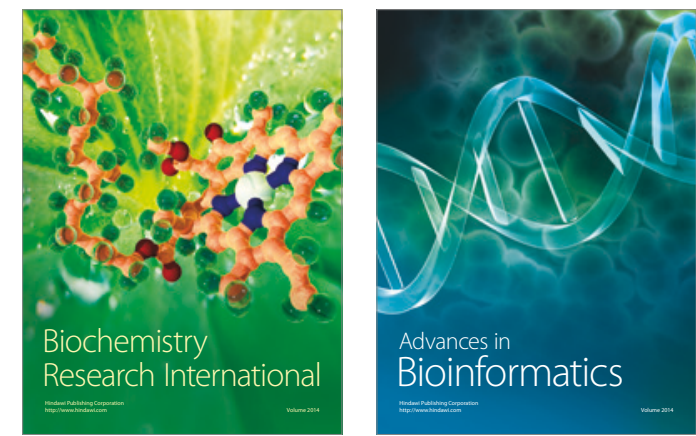

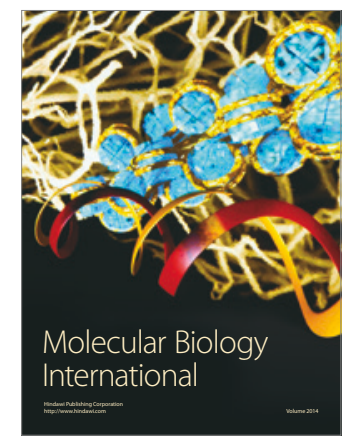

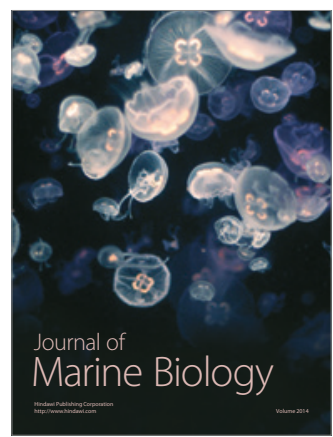

